\title{
Post Main-Sequence Changes in Rotational Velocities
}

\author{
Helmut A. Abt \\ Kitt Peak National Observatory, Box 26732, Tucson, AZ 85726-6732, \\ $U S A$
}

\begin{abstract}
From recent measurements of the rotational velocities of $1100 \mathrm{~B}$ stars and 1700 A stars, we determined the maximum periods of binaries with fully circularized orbits. Combined with published data, we derived the relation of period (days) $=0.001$ age $^{0.42}(\mathrm{yr})$. We were able to reproduce the rotational velocities of giants from $v \sin i$ of dwarfs only if rigid body rotation applies to both. However for evolutionary expansions greater than 5 the angular momentum is conserved in shells, which is reasonable physically.
\end{abstract}

\section{1. Introduction}

New projected rotational velocities, $v \sin i$, were derived for about $1100 \mathrm{~B}$ stars by Abt, Levato, \& Grosso (2002) and for 1700 A stars by Abt \& Morrell (1995). Those showed that for stars in binaries, all the B primaries with periods $<2.4$ days are fully synchronized and for A primaries with periods $<4.9$ days. These are consistent with the theoretical results of Zahn (1977). Further, all B stars with periods $<1.5$ have circular orbits and all $\mathrm{A}$ stars with periods $<2.5$ days are circular. Combining these data for Hyades \& Praesepe and old disk stars (Duquennoy \& Mayor 1991), M67 (Mathieu \& Mazeh 1988), and halo stars (Latham et al. 1988), we find the linear relation circularized $P$ (days) $=0.001$ age $^{0.42}(\mathrm{yr})$.

\subsection{Post Main-Sequence Rotational Velocities}

Using the mean projected rotational velocities of $1377 \mathrm{~B}$ and $\mathrm{A}$ dwarfs (normal and peculiar), we attempt to explain the mean rotational velocities of $381 \mathrm{~B}$ and A giants. We assumed evolutionary models of Bertelli et al. (1986) and assumed a mean MS age for the dwarfs of $30 \times 10^{6} \mathrm{yr}$. The data do not allow any significant loss of angular momentum with either extreme transfer method. The extreme transfer methods are a free transfer of angular momentum, i.e. continuous rigid body rotation, or no transfer of angular momentum, i.e. conservation in shells. Conservation in shells does not fit the data but a free transfer of angular momentum within the stars fit within the measuring errors of $(15 \mathrm{~km}$ $\mathrm{s}^{-1}$. This confirms the results of Oke \& Greenstein (1954) and Sandage (1955) for late-type giants that have expanded by less than a factor of about 5 from the main sequence. However, Abt $(1957,1958)$ found that the rotational velocities of bright giants and supergiants that have expanded by factors of 4-10 from dwarfs can be understood only with conservation in shells. Thus conservation in rigid-body rotation applies for expansions $<5$ and in shells for expansions of 510. This conclusion seems reasonable in view of the gradual density gradients in 
dwarfs but in supergiants the interior structure consists of a small high-density core and a very tenuous envelope. We would not expect much coupling of envelope and core in the latter case. Another example is the atmosphere of the $\mathrm{K}$ bright giant in zeta Aurigae. Wilson \& Abt (1954) could explain why the UV radiation of the $B$ stars did not ionize the atmosphere of the $K$ star only if the atmosphere of the $\mathrm{K}$ stars consisted of high density condensations (sheets, clumps, or prominences) of order $10^{3} \mathrm{~km}$. The rotational motions of such condensations are unlikely to be coupled with the stellar core.

\section{References}

Abt, H. A. 1957, ApJ 126, 503

Abt, H. A. 1958. ApJ 127, 658

Abt, H. A., Levato, H., \& Grosso, M. 2002, ApJ 573, 359

Abt, H. A., \& Morrell, N. I. 1995, ApJS, 99, 135

Bertelli, G., Bressan, A., Chiosi, C., \& Angerer, K. 1986, A\&A 66, 191

Duquennoy, A., \& Mayor, M. 1991, A\&A 248, 485

Latham, D. W., Mazeh, T., Carney, B. W., McCrosky, R. E., Stefanik, R. P., \& Davis, R. J. 1988, 567

Mathieu, R. D., \& Mazeh, T. 1988, ApJ 326, 256

Oke, J. B., \& Greenstein, J. L. 1954, ApJ 120, 384

Sandage, A. 1955, ApJ 122, 263

Wilson, O. C., \& Abt, H. A. 1954, ApJS 1, 1

Zahn, J. P. 1977, A\&A 57, 383

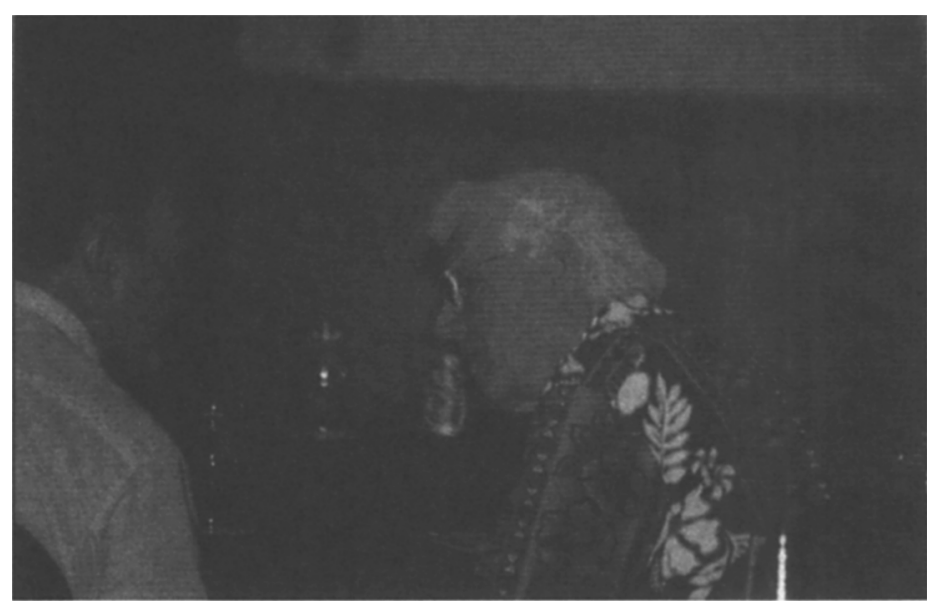

Jean Zorec and Helmut Abt discussing about maybe rotation. 